\title{
Predictive value of peripheral regulatory $T$ cells in non-small cell lung cancer patients undergoing radiotherapy
}

\author{
Chao Liu ${ }^{1}$, Shikai Wu ${ }^{1}$, Xiangying Meng ${ }^{1}$, Guangxian Liu ${ }^{2}$, Dongmei Chen ${ }^{1}$, Yang \\ Cong ${ }^{1}$, Ge Shen ${ }^{1}$, Bing Sun ${ }^{1}$, Wei Wang ${ }^{2}$, Qian Wang ${ }^{1}$, Hongjun Gao $^{3}$ and Xiaoqing \\ Liu $^{3}$ \\ ${ }^{1}$ Department of Radiation Oncology, Affiliated Hospital of Academy of Military Medical Sciences, Beijing, China \\ ${ }^{2}$ Cancer Therapy Center, Affiliated Hospital of Academy of Military Medical Sciences, Beijing, China \\ ${ }^{3}$ Department of Lung Cancer, Affiliated Hospital of Academy of Military Medical Sciences, Beijing, China \\ Correspondence to: Shikai Wu, email: skywu4923@sina.com
}

Keywords: T cells; lymphocyte subsets; stereotactic body radiation therapy; non-small cell lung cancer; immunotherapy Received: December 19,2016 Accepted: January 27, $2017 \quad$ Published: February 09, 2017

Copyright: Liu et al. This is an open-access article distributed under the terms of the Creative Commons Attribution License 3.0 (CC BY 3.0), which permits unrestricted use, distribution, and reproduction in any medium, provided the original author and source are credited.

\section{ABSTRACT}

Background: Studies increasingly focus on the impact of radiotherapy on immunity; however, the role of peripheral cellular immunity prior to radiotherapy in cancer patients remains largely unknown. In this study, we investigated the predictive roles of lymphocyte subsets on tumor progression in non-small cell lung cancer (NSCLC) patients undergoing radiotherapy, and their expression in NSCLC patients at first relapse.

Methods: We enrolled 70 NSCLC patients and 14 age- and sex-matched healthy donors and tested the lymphocyte subsets in their peripheral blood by flow cytometry. Among them, $\mathbf{4 0}$ newly diagnosed patients received radiotherapy and were enrolled to investigate the predictive value of lymphocyte subsets on tumor progression after radiotherapy by uni- and multivariate analyses; $\mathbf{3 0}$ patients at first relapse were included to evaluate the differences of lymphocyte subsets between them and first diagnosed patients and healthy volunteers.

Results: Increased proportions of regulatory $T$ cells, CD8 $+T$ cells, and CD8+CD28- $T$ cells and decreased CD4+ $T$ cells and CD4/CD8 ratios were observed in NSCLC patients at first relapse compared to newly diagnosed patients. In the 40 first diagnosed patients undergoing radiotherapy, uni- and multivariate analyses showed that increased level of regulatory $\mathrm{T}$ cells correlated with poor progression-free survival (hazard ratio $=2.55$ and $3.76, P=0.022$ and 0.010 , respectively).

Conclusions: Peripheral regulatory $\mathrm{T}$ cells were increased and independently predict tumor progression in NSCLC patients undergoing radiotherapy, suggesting the promising combination of radiotherapy and immunotherapy.

\section{INTRODUCTION}

As the most common malignant tumor worldwide, approximately $80 \%$ of lung cancer patients are nonsmall cell lung cancer (NSCLC) cases $[1,2]$. In recent years, radiotherapy lonely and in combination with other therapies are used as prevailing treatments in $\sim 60 \%$ of newly diagnosed NSCLC patients [3]. Stereotactic body radiotherapy (SBRT) is becoming as the predominant treatment for NSCLC, especially for early stage ones [4, 5]. Besides the effect of direct killing, radiation induces immunologic response and consequently eliminates nonirradiated tumor cells $[6,7]$. According to the immune mechanism of radiotherapy, we proposed that the immune status before radiotherapy may impact the tumor response to radiation and accordingly affect long-term survival. Thus, we aimed to evaluate the clinical values of pretreatment immune cells in peripheral blood in NSCLC patients undergoing radiation therapy. 
The theory of cancer immunoediting, including immune elimination, equilibrium, and escape, describes the dual role of immunity in tumor suppression and protection. Immune function in cancer patients closely relates to cancer occurrence and progression [8]. Lymphocytes are significant components of human cellular immunity; different lymphocyte subsets have been observed according to different phenotypes and functions [9]. Several studies have investigated the expression of peripheral lymphocyte subsets in NSCLC. For example, decreased proportions of CD4+ cells ( $\mathrm{T}$ helper cells), CD4/CD8 ratios, and B cells and increased CD8+CD28- T lymphocytes and regulatory $\mathrm{T}$ (Treg) cells were observed in patients with lung cancer [10-13].

In addition to the expression of lymphocyte subsets, researchers also tested their predictive and prognostic roles in NSCLC. Many studies proved the prognostic value of tumor-infiltrating lymphocytes of different subtypes, including CD3+, CD4+, CD8+, Treg cells [14-18]. However, the predictive roles of lymphocyte subpopulations in peripheral blood were not well studied. McCoy et al. [19] found that an elevated proportion of peripheral Treg cells was associated with poor survival in patients with thoracic malignancies. A very recent study has demonstrated that the prognostic value of CD4+ Treg subtypes in NSCLC patients received chemotherapy [20].
Nevertheless, in NSCLC patients undergoing radiotherapy, the clinical significance of lymphocyte subsets is poorly investigated.

The present study aimed to evaluate the predictive value of Treg cells and other lymphocyte subsets in NSCLC patients treated with radiotherapy, and their expression in NSCLC patients at first relapse.

\section{RESULTS}

\section{Baseline characteristics}

Table 1 shows the clinicopathologic characteristics of 70 NSCLC patients. The mean age of 70 NSCLC patients was 63 (range 36-90). No differences of baseline characteristics were observed between patients at first diagnosis and relapse, providing an opportunity to compare the expression of lymphocyte subsets between them. Among 40 newly diagnosed patients, 24 (60\%) were treated with SBRT with 30-50 Gy/5 fractions; 16 $(40 \%)$ received conventional fraction radiotherapy with 60-66 Gy/30-33 fractions for their lung masses. Twentytwo patients underwent positron emission tomographycomputed tomography before treatment (data not shown);
A

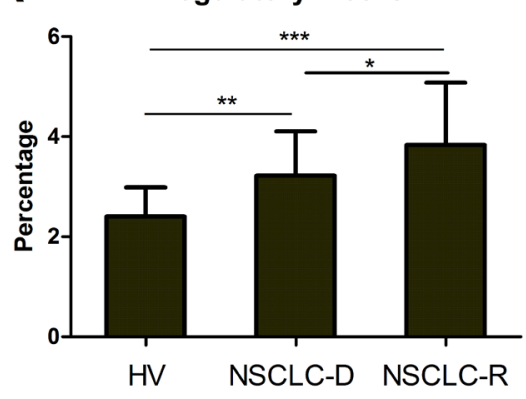

D

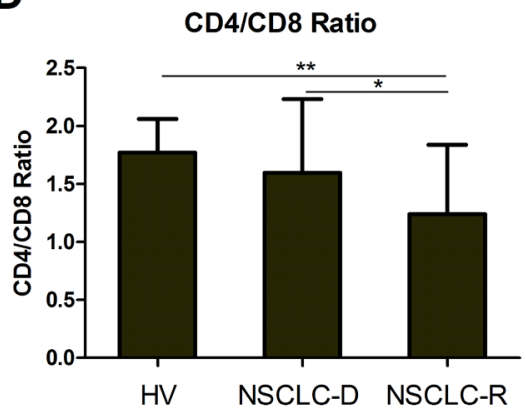

B

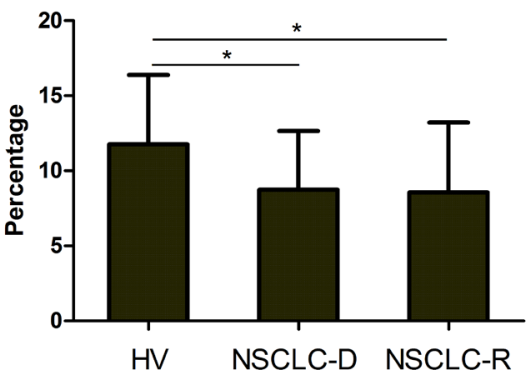

E

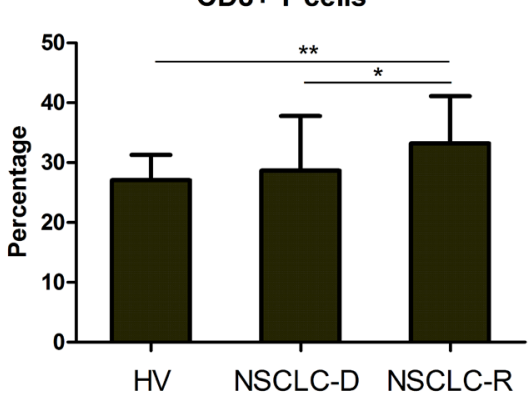

C

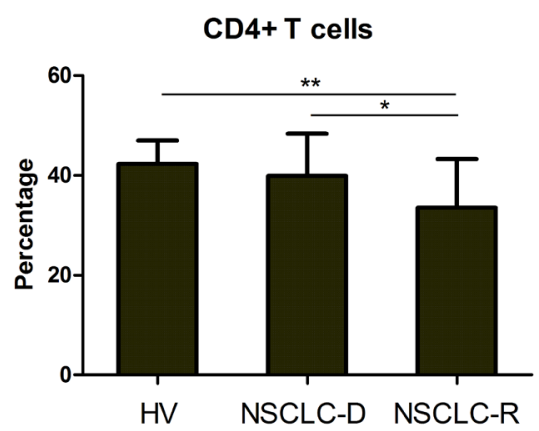

$\mathbf{F}$

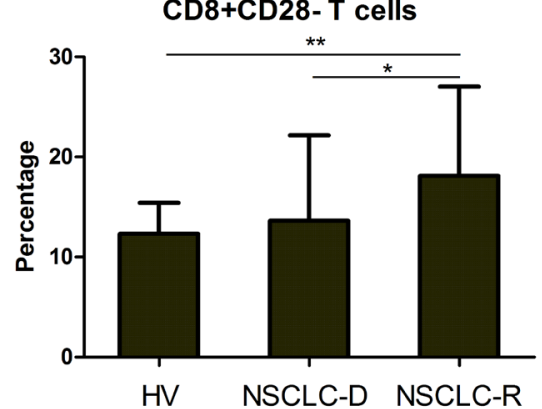

Figure 1: The proportions of lymphocyte subsets in NSCLC patients. A. Increased regulatory T cells in NSCLC-D and NSCLC-R; Decreased B. CD19+ B cells, C. CD4+ T cells, and D. CD4/CD8 ratio; Elevated E. CD8+ T cells and F. CD8+CD28- T cells. Abbreviations: NSCLC $=$ non-small cell lung cancer; HV = healthy volunteers; NSCLC-D = NSCLC-at first diagnosis; NSCLC-R = NSCLC-at first relapse. $* P<0.05 ; * * P<0.01 ; * * * P<0.001$. 
Table 1: Comparisons of baseline characteristics between NSCLC-D and NSCLC-R

\begin{tabular}{|c|c|c|c|}
\hline \multicolumn{4}{|c|}{ NSCLC Patients $(N=70)$} \\
\hline \multirow[t]{2}{*}{ Characteristic } & NSCLC-D $(N=40)$ & NSCLC-R $(N=30)$ & $P$ \\
\hline & $N(\%)$ & $N(\%)$ & \\
\hline \multicolumn{4}{|l|}{ Sex } \\
\hline Male & $28(70)$ & $22(73.3)$ & 0.760 \\
\hline Female & $12(30)$ & $8(26.7)$ & \\
\hline \multicolumn{4}{|l|}{ Age(years) } \\
\hline$<60$ & $14(35)$ & $15(50)$ & 0.207 \\
\hline$\geq 60$ & $26(65)$ & $15(50)$ & \\
\hline \multicolumn{4}{|l|}{ Smoking } \\
\hline Never & $14(35)$ & $11(36.7)$ & 0.688 \\
\hline Previous & $3(7.5)$ & $4(13.3)$ & \\
\hline Present & $23(57.5)$ & $15(50)$ & \\
\hline \multicolumn{4}{|l|}{ ECOG PS } \\
\hline 0 & $16(40)$ & $10(33.3)$ & 0.669 \\
\hline 1 & $21(52.5)$ & $19(63.3)$ & \\
\hline 2 & $3(7.5)$ & $1(3.3)$ & \\
\hline \multicolumn{4}{|l|}{ Histology } \\
\hline $\mathrm{SCC}$ & $19(47.5)$ & $15(50)$ & 0.929 \\
\hline ADC & $19(47.5)$ & $13(43.3)$ & \\
\hline Other & $2(5)$ & $2(6.7)$ & \\
\hline \multicolumn{4}{|l|}{ Differentiation } \\
\hline Well & $3(7.5)$ & $2(6.7)$ & 0.925 \\
\hline Moderate & $21(52.5)$ & $15(50)$ & \\
\hline Poor & $9(22.5)$ & $9(30)$ & \\
\hline Unknown & $7(17.5)$ & $4(13.3)$ & \\
\hline \multicolumn{4}{|l|}{ Gene mutation } \\
\hline Yes & $5(12.5)$ & $5(16.7)$ & 0.906 \\
\hline No & $32(80)$ & $23(76.7)$ & \\
\hline Unknown & $3(7.5)$ & $2(6.7)$ & \\
\hline \multicolumn{4}{|l|}{ Tumor stage } \\
\hline T1 & $10(25)$ & $6(20)$ & 0.835 \\
\hline T2 & $17(42.5)$ & $12(40)$ & \\
\hline T3 & $6(15)$ & $7(23.3)$ & \\
\hline T4 & $7(17.5)$ & $5(16.7)$ & \\
\hline \multicolumn{4}{|l|}{ Nodal stage } \\
\hline N0 & $11(27.5)$ & $7(23.3)$ & 0.639 \\
\hline N1 & $11(27.5)$ & $7(23.3)$ & \\
\hline $\mathrm{N} 2$ & $10(25)$ & $12(40)$ & \\
\hline $\mathrm{N} 3$ & $8(20)$ & $4(13.3)$ & \\
\hline \multicolumn{4}{|l|}{ Metastasis } \\
\hline Yes & $11(27.5)$ & $9(30)$ & 0.819 \\
\hline No & $29(72.5)$ & $21(70)$ & \\
\hline \multicolumn{4}{|l|}{ AJCC stage } \\
\hline 1 & $4(10)$ & $5(16.7)$ & 0.791 \\
\hline 2 & $8(20)$ & $4(13.3)$ & \\
\hline 3 & $17(42.5)$ & $12(40)$ & \\
\hline 4 & $11(27.5)$ & $9(30)$ & \\
\hline
\end{tabular}

Abbreviations: NSCLC: non-small cell lung cancer; NSCLC-D: NSCLC-at first diagnosis; NSCLC-R: NSCLC-at first relapse; ECOG PS: Eastern Cooperative Oncology Group performance status; SCC: squamous cell carcinoma; ADC: adenocarcinoma; AJCC: American Joint Committee on Cancer. 
Table 2: Comparisons of lymphocyte subsets between NSCLC patients and healthy controls

\begin{tabular}{|l|c|c|c|c|}
\hline \multicolumn{1}{|c|}{ Immune parameter } & Healthy control & NSCLC-D & NSCLC-R & $\boldsymbol{P}$ \\
\hline $\mathrm{CD} 3+\mathrm{T}$ cells & $72.87 \pm 5.40$ & $69.63 \pm 12.83$ & $67.89 \pm 11.02$ & 0.398 \\
\hline $\mathrm{CD} 4+\mathrm{T}$ cells & $42.26 \pm 4.70$ & $39.85 \pm 8.49$ & $33.53 \pm 9.74$ & $\mathbf{0 . 0 0 2}$ \\
\hline $\mathrm{CD} 8+\mathrm{T}$ cells & $27.05 \pm 4.21$ & $28.65 \pm 9.12$ & $33.20 \pm 7.00$ & $\mathbf{0 . 0 2 5}$ \\
\hline $\mathrm{CD} 4 / \mathrm{CD} 8$ ratio & $1.77 \pm 0.29$ & $1.60 \pm 0.63$ & $1.24 \pm 0.60$ & $\mathbf{0 . 0 0 8}$ \\
\hline $\mathrm{CD} 8+\mathrm{CD} 28+\mathrm{T}$ cells & $14.59 \pm 2.70$ & $13.72 \pm 4.92$ & $13.44 \pm 3.86$ & 0.708 \\
\hline $\mathrm{CD} 8+\mathrm{CD} 28-\mathrm{T}$ cells & $12.32 \pm 3.10$ & $13.64 \pm 8.52$ & $18.11 \pm 8.92$ & $\mathbf{0 . 0 3 3}$ \\
\hline Treg & $2.40 \pm 0.59$ & $3.22 \pm 0.89$ & $3.83 \pm 1.24$ & $\mathbf{0 . 0 0 1}$ \\
\hline $\mathrm{CD} 19+\mathrm{B}$ cells & $11.77 \pm 4.62$ & $8.74 \pm 3.91$ & $8.57 \pm 4.66$ & 0.058 \\
\hline $\mathrm{NK}$ & $16.24 \pm 6.33$ & $20.22 \pm 11.92$ & $20.98 \pm 8.91$ & 0.350 \\
\hline $\mathrm{NKT}$ & $6.85 \pm 4.20$ & $5.54 \pm 4.63$ & $6.37 \pm 4.22$ & 0.590 \\
\hline$\gamma \delta \mathrm{T}$ & $5.62 \pm 4.66$ & $5.17 \pm 7.08$ & $5.11 \pm 4.42$ & 0.963 \\
\hline
\end{tabular}

Data were expressed as Mean \pm SD. Abbreviations: NSCLC: non-small cell lung cancer; NSCLC-D: NSCLC-at first diagnosis; NSCLC-R: NSCLC-at first relapse; SD: standard deviation; Treg: regulatory T cells.

Table 3: Differences of lymphocyte subsets between local recurrence and metastasis in 30 NSCLC patients at first relapse

\begin{tabular}{|l|c|c|c|}
\hline \multicolumn{1}{|c|}{ Immune parameter } & Local recurrence $(\boldsymbol{N}=\mathbf{1 8})$ & Metastasis $(\boldsymbol{N = 1 2})$ & $\boldsymbol{P}$ \\
\hline CD3+ T cells & $68.72 \pm 11.03$ & $66.63 \pm 11.36$ & 0.619 \\
\hline CD4+ T cells & $37.42 \pm 11.18$ & $32.14 \pm 9.08$ & 0.184 \\
\hline CD8+ T cells & $30.20 \pm 8.51$ & $32.71 \pm 7.56$ & 0.416 \\
\hline CD4/CD8 ratio & $1.38 \pm 0.69$ & $1.03 \pm 0.37$ & 0.083 \\
\hline CD8+CD28+ T cells & $13.09 \pm 4.10$ & $13.97 \pm 3.56$ & 0.548 \\
\hline CD8+CD28- T cells & $17.52 \pm 9.83$ & $19.00 \pm 7.70$ & 0.665 \\
\hline Treg & $2.61 \pm 0.98$ & $3.55 \pm 1.21$ & $\mathbf{0 . 0 4 6}$ \\
\hline CD19+ B cells & $8.02 \pm 3.66$ & $9.26 \pm 5.82$ & 0.547 \\
\hline NK & $19.66 \pm 8.90$ & $22.65 \pm 9.06$ & 0.418 \\
\hline NKT & $6.36 \pm 5.34$ & $6.38 \pm 2.39$ & 0.990 \\
\hline$\gamma \delta T$ & $4.97 \pm 4.45$ & $5.29 \pm 4.60$ & 0.864 \\
\hline
\end{tabular}

Data were expressed as Mean \pm SD. Abbreviations: NSCLC: non-small cell lung cancer; SD: standard deviation; Treg: regulatory T cells.

mean standard uptake value (SUV) of lung mass was 11.1 (range, 3.2-30.8). There were 3 patients with epidermal growth factor receptor mutation and 2 patients with anaplastic lymphoma kinase gene fusion and KRAS mutation, respectively.

\section{The proportions of lymphocyte subsets in NSCLC patients}

The percentage of Treg cells in cancer patients were considerably elevated compared to healthy controls; the high expression of Treg cells was further validated in NSCLC patients at first relapse compared to newly diagnosed patients (Table 2, Figure 1A). In contrast, decreased proportions of CD19+ B cells, CD4+ T cells, and $\mathrm{CD} 4 / \mathrm{CD} 8$ ratios were observed (Table 2, Figure 1B1D). Besides, increased CD8+ T cells and CD8+CD28- T cells were showed in NSCLC patients (Table 2, Figure $2 \mathrm{E}-2 \mathrm{~F}$ ). However, there were no differences in NK, NKT, $\gamma \delta \mathrm{T}, \mathrm{CD} 3+$ and CD8+CD28+ T cells between patients and controls (Table 2). In 30 patients at first relapse, increased Treg cells were observed in metastatic patients compared to recurrent patients; decreased proportions of CD4+ T cells, CD4/CD8 ratios were presented with a statistical trend (Table 3 ).

\section{Correlation of lymphocyte subpopulations with clinicopathologic parameters}

Table 4-5 summarizes the associations between lymphocyte subsets and clinicopathological variables in 40 newly diagnosed patients. Patients who were younger and with negative tumor markers had higher proportions of CD3 + T cells compared to their counterparts. Similarly, increased CD4+ T cells were observed in young patients. In females, there were elevated proportions of CD4+ $\mathrm{T}$ cells and CD4/CD8 ratios compared to males. High levels of $\mathrm{CD} 8+\mathrm{CD} 28+\mathrm{T}$ cells were occurred in patients with 
Table 4: Correlation between $T$ lymphocyte subsets and clinicopathologic characteristics in 40 newly diagnosed NSCLC patients

\begin{tabular}{|c|c|c|c|c|c|c|c|c|c|c|c|c|c|c|}
\hline Parameter & CD3 $+T$ cells & $P$ & $\begin{array}{|lr|}\begin{array}{l}\text { CD4+ } \\
\text { cells }\end{array} & \text { T } \\
\end{array}$ & $P$ & CD8+ $T$ cells & $P$ & \begin{tabular}{|l|} 
CD4/CD8 \\
ratio
\end{tabular} & $P$ & $\begin{array}{l}\text { CD8+CD28+T } \\
\text { cells }\end{array}$ & $P$ & $\begin{array}{l}\text { CD8+CD28- } \\
\text { T cells }\end{array}$ & $P$ & Treg & $P$ \\
\hline \multicolumn{15}{|l|}{ Sex } \\
\hline Female & $72.86 \pm 12.42$ & 0.303 & $44.04 \pm 8.04$ & 0.046 & $26.64 \pm 12.39$ & 0.749 & $2.11 \pm 1.30$ & 0.036 & $12.41 \pm 3.42$ & 0.277 & $13.55 \pm 9.26$ & 0.965 & $3.42 \pm 1.20$ & 0.562 \\
\hline Male & $68.24 \pm 12.97$ & & $37.28 \pm 10.01$ & & $27.73 \pm 8.60$ & & $1.47 \pm 0.58$ & & $14.28 \pm 5.40$ & & $13.68 \pm 8.37$ & & $3.20 \pm 0.93$ & \\
\hline \multicolumn{15}{|l|}{ Age(years) } \\
\hline$<60$ & $75.11 \pm 10.36$ & 0.046 & $44.03 \pm 7.74$ & 0.024 & $28.65 \pm 10.00$ & 0.559 & $1.72 \pm 0.67$ & 0.769 & $14.19 \pm 3.95$ & 0.662 & $13.43 \pm 8.19$ & 0.909 & $3.45 \pm 1.18$ & 0.414 \\
\hline$\geq 60$ & $66.68 \pm 13.24$ & & $36.76 \pm 10.08$ & & $26.73 \pm 9.73$ & & $1.63 \pm 1.00$ & & $13.47 \pm 5.43$ & & $13.76 \pm 8.86$ & & $3.16 \pm 0.89$ & \\
\hline \multicolumn{15}{|l|}{ Smoking } \\
\hline Never & $71.78 \pm 12.19$ & 0.445 & $40.15 \pm 10.07$ & 0.697 & $26.37 \pm 11.80$ & 0.628 & $1.92 \pm 1.24$ & 0.180 & $10.71 \pm 3.15$ & 0.003 & $15.51 \pm 10.83$ & 0.315 & $3.06 \pm 1.05$ & 0.426 \\
\hline $\begin{array}{l}\text { Previous/ } \\
\text { present }\end{array}$ & $68.47 \pm 13.25$ & & $38.85 \pm 9.94$ & & $27.96 \pm 8.64$ & & $1.53 \pm 0.62$ & & $15.34 \pm 4.99$ & & $12.63 \pm 7.03$ & & $3.35 \pm 0.98$ & \\
\hline \multicolumn{15}{|l|}{ ECOG PS } \\
\hline 0 & $72.34 \pm 10.66$ & 0.281 & $40.34 \pm 7.51$ & 0.597 & $29.51 \pm 9.59$ & 0.269 & $1.65 \pm 1.13$ & 0.928 & $14.87 \pm 5.04$ & 0.233 & $15.32 \pm 7.83$ & 0.314 & $3.29 \pm 0.63$ & 0.919 \\
\hline $1-2$ & $67.82 \pm 14.02$ & & $38.62 \pm 11.28$ & & $26.00 \pm 9.78$ & & $1.68 \pm 0.72$ & & $12.96 \pm 4.80$ & & $12.52 \pm 8.94$ & & $3.25 \pm 1.17$ & \\
\hline \multicolumn{15}{|l|}{ SUV } \\
\hline$<9.5$ & $69.51 \pm 14.92$ & 0.949 & $39.21 \pm 9.44$ & 0.479 & $27.68 \pm 10.70$ & 0.982 & $1.76 \pm 1.36$ & 0.582 & $12.96 \pm 4.21$ & 0.364 & $14.85 \pm 8.32$ & 0.557 & $3.00 \pm 0.90$ & 0.600 \\
\hline$\geq 9.5$ & $69.11 \pm 14.22$ & & $36.00 \pm 11.09$ & & $27.58 \pm 10.73$ & & $1.51 \pm 0.68$ & & $11.30 \pm 4.18$ & & $17.38 \pm 10.98$ & & $2.81 \pm 0.66$ & \\
\hline \multicolumn{15}{|l|}{ Histology } \\
\hline SCC & $66.76 \pm 15.13$ & 0.228 & $36.29 \pm 10.57$ & 0.138 & $27.33 \pm 9.87$ & 0.779 & $1.48 \pm 0.60$ & 0.366 & $13.48 \pm 4.90$ & 0.711 & $14.21 \pm 9.81$ & 0.866 & $3.05 \pm 0.97$ & 0.422 \\
\hline $\mathrm{ADC}$ & $71.92 \pm 10.37$ & & $40.88 \pm 7.89$ & & $28.24 \pm 10.07$ & & $1.74 \pm 1.09$ & & $14.10 \pm 5.30$ & & $13.72 \pm 7.53$ & & $3.31 \pm 0.91$ & \\
\hline \multicolumn{15}{|c|}{ Differentiation } \\
\hline $\begin{array}{l}\text { Well/ } \\
\text { moderate }\end{array}$ & $69.9 \pm 12.63$ & 0.675 & $39.34 \pm 8.67$ & 0.170 & $27.94 \pm 10.41$ & 0.828 & $1.68 \pm 0.98$ & 0.256 & $12.78 \pm 3.96$ & 0.234 & $14.64 \pm 9.12$ & 0.903 & $2.95 \pm 0.84$ & 0.397 \\
\hline Poor & $67.6 \pm 17.16$ & & $34.07 \pm 11.89$ & & $28.80 \pm 9.12$ & & $1.27 \pm 0.59$ & & $14.98 \pm 6.21$ & & $15.08 \pm 8.76$ & & $3.25 \pm 0.91$ & \\
\hline \multicolumn{15}{|c|}{ Tumor stage } \\
\hline $\mathrm{T} 1$ & $65.48 \pm 12.86$ & 0.243 & $37.48 \pm 8.89$ & 0.507 & $25.27 \pm 9.61$ & 0.430 & $1.83 \pm 1.34$ & 0.519 & $12.05 \pm 5.71$ & 0.221 & $13.94 \pm 6.40$ & 0.899 & $3.10 \pm 0.70$ & 0.601 \\
\hline $\mathrm{T} 2-\mathrm{T} 4$ & $71.01 \pm 12.74$ & & $39.92 \pm 10.25$ & & $28.12 \pm 9.84$ & & $1.61 \pm 0.71$ & & $14.28 \pm 4.61$ & & $13.54 \pm 9.22$ & & $3.31 \pm 1.07$ & \\
\hline \multicolumn{15}{|c|}{ Nodal stage } \\
\hline N0 & $69.61 \pm 10.82$ & 0.995 & $39.02 \pm 9.23$ & 0.913 & $27.93 \pm 10.76$ & 0.836 & $1.77 \pm 1.34$ & 0.654 & $14.71 \pm 4.84$ & 0.443 & $12.60 \pm 6.55$ & 0.641 & $3.24 \pm 1.01$ & 0.936 \\
\hline N1-N3 & $69.64 \pm 13.70$ & & $39.41 \pm 10.26$ & & $27.20 \pm 9.52$ & & $1.63 \pm 0.68$ & & $13.35 \pm 4.99$ & & $14.03 \pm 9.24$ & & $3.27 \pm 1.01$ & \\
\hline \multicolumn{15}{|c|}{ AJCC stage } \\
\hline $1-3$ & $68.79 \pm 12.97$ & 0.508 & $38.83 \pm 10.07$ & 0.626 & $26.90 \pm 9.39$ & 0.598 & $1.67 \pm 0.94$ & 0.950 & $13.43 \pm 4.49$ & 0.555 & $13.61 \pm 8.35$ & 0.974 & $3.21 \pm 0.98$ & 0.614 \\
\hline 4 & $71.85 \pm 12.79$ & & $40.56 \pm 9.69$ & & $28.75 \pm 10.96$ & & $1.65 \pm 0.80$ & & $14.48 \pm 6.11$ & & $13.71 \pm 9.38$ & & $3.39 \pm 1.07$ & \\
\hline \multicolumn{15}{|c|}{ Tumor Marker } \\
\hline Negative & $75.53 \pm 8.82$ & 0.049 & $41.98 \pm 6.86$ & 0.241 & $32.44 \pm 9.88$ & 0.026 & $1.46 \pm 0.68$ & 0.294 & $15.91 \pm 5.14$ & 0.050 & $15.58 \pm 8.00$ & 0.288 & $3.27 \pm 1.18$ & 0.960 \\
\hline Positive & $66.41 \pm 13.64$ & & $38.43 \pm 10.82$ & & $24.62 \pm 9.13$ & & $1.81 \pm 0.98$ & & $12.45 \pm 4.56$ & & $12.27 \pm 8.75$ & & $3.25 \pm 0.97$ & \\
\hline
\end{tabular}

Data were expressed as Mean \pm SD. Abbreviations: NSCLC: non-small cell lung cancer; SD: standard deviation; Treg: regulatory T cells; SUV: standard uptake value; ECOG PS: Eastern Cooperative Oncology Group performance status; SCC: squamous cell carcinoma; ADC: adenocarcinoma; AJCC: American Joint Committee on Cancer.

negative tumor markers (Table 4). Nonsmokers showed increased $\gamma \delta$ T cells compared to smokers (Table 5). We found no significant associations between $\mathrm{CD} 8+\mathrm{CD} 28$ T cells, Treg, CD19+ B cells, NK, NKT cells and clinicopathologic characteristics.

\section{Increased Treg cells correlated with poor progression-free survival (PFS)}

Table 6 summarizes the results of univariate analysis of lymphocyte subsets. High proportions of Treg cells correlated to worse PFS $(\mathrm{HR}=2.55,95 \% \mathrm{CI}=1.07$ $6.11, P=0.022$, Figure 2A). We found no significant 
Table 5: Correlation between other lymphocyte subsets and clinicopathologic characteristics in 40 newly diagnosed NSCLC patients

\begin{tabular}{|c|c|c|c|c|c|c|c|c|}
\hline Parameter & CD19+ B cells & $P$ & NK & $P$ & NKT & $P$ & $\gamma \delta \mathrm{T}$ & $P$ \\
\hline \multicolumn{9}{|l|}{ Sex } \\
\hline Female & $11.72 \pm 6.13$ & 0.057 & $14.49 \pm 10.65$ & 0.073 & $4.53 \pm 2.81$ & 0.423 & $4.13 \pm 2.61$ & 0.590 \\
\hline Male & $8.36 \pm 3.90$ & & $22.43 \pm 11.83$ & & $5.93 \pm 5.16$ & & $5.58 \pm 8.19$ & \\
\hline \multicolumn{9}{|l|}{ Age(years) } \\
\hline$<60$ & $9.54 \pm 5.90$ & 0.823 & $15.74 \pm 10.05$ & 0.090 & $5.71 \pm 2.92$ & 0.873 & $5.14 \pm 3.56$ & 0.986 \\
\hline$\geq 60$ & $9.16 \pm 4.15$ & & $22.75 \pm 12.35$ & & $5.45 \pm 5.43$ & & $5.19 \pm 8.53$ & \\
\hline \multicolumn{9}{|l|}{ Smoking } \\
\hline Never & $9.60 \pm 6.39$ & 0.802 & $17.54 \pm 10.62$ & 0.377 & $6.70 \pm 7.12$ & 0.330 & $9.60 \pm 11.59$ & 0.011 \\
\hline Previous/present & $9.16 \pm 4.03$ & & $21.41 \pm 12.47$ & & $5.04 \pm 3.06$ & & $3.23 \pm 2.04$ & \\
\hline \multicolumn{9}{|l|}{ ECOG PS } \\
\hline 0 & $8.22 \pm 2.63$ & 0.318 & $18.31 \pm 10.13$ & 0.476 & $4.24 \pm 2.61$ & 0.211 & $4.09 \pm 2.26$ & 0.498 \\
\hline $1-2$ & $9.90 \pm 5.61$ & & $21.31 \pm 12.91$ & & $6.28 \pm 5.38$ & & $5.79 \pm 8.71$ & \\
\hline \multicolumn{9}{|l|}{ SUV } \\
\hline$<9.5$ & $7.84 \pm 3.59$ & 0.728 & $22.00 \pm 15.39$ & 0.854 & $4.11 \pm 1.70$ & 0.145 & $4.71 \pm 2.61$ & 0.427 \\
\hline$\geq 9.5$ & $7.25 \pm 3.60$ & & $20.89 \pm 10.41$ & & $8.08 \pm 7.60$ & & $8.26 \pm 12.82$ & \\
\hline \multicolumn{9}{|l|}{ Histology } \\
\hline $\mathrm{SCC}$ & $8.45 \pm 3.77$ & 0.435 & $23.58 \pm 12.86$ & 0.138 & $5.58 \pm 6.09$ & 0.933 & $5.43 \pm 9.49$ & 0.865 \\
\hline $\mathrm{ADC}$ & $9.52 \pm 4.14$ & & $17.41 \pm 10.46$ & & $5.72 \pm 2.72$ & & $5.00 \pm 3.82$ & \\
\hline \multicolumn{9}{|l|}{ Differentiation } \\
\hline Well/moderate & $8.83 \pm 3.40$ & 0.579 & $19.86 \pm 10.23$ & 0.455 & $5.26 \pm 5.28$ & 0.364 & $5.68 \pm 8.67$ & 0.892 \\
\hline Poor & $7.92 \pm 5.28$ & & $23.80 \pm 17.87$ & & $7.15 \pm 3.81$ & & $5.24 \pm 4.15$ & \\
\hline \multicolumn{9}{|l|}{ Tumor stage } \\
\hline $\mathrm{T} 1$ & $9.32 \pm 3.80$ & 0.989 & $24.93 \pm 11.68$ & 0.210 & $4.09 \pm 1.84$ & 0.323 & $3.88 \pm 3.48$ & 0.564 \\
\hline $\mathrm{T} 2-\mathrm{T} 4$ & $9.29 \pm 5.09$ & & $18.88 \pm 11.85$ & & $5.96 \pm 5.11$ & & $5.54 \pm 7.82$ & \\
\hline \multicolumn{9}{|l|}{ Nodal stage } \\
\hline No & $9.04 \pm 3.45$ & 0.860 & $18.96 \pm 10.72$ & 0.719 & $5.11 \pm 2.52$ & 0.751 & $3.67 \pm 2.63$ & 0.470 \\
\hline N1-N3 & $9.38 \pm 5.20$ & & $20.65 \pm 12.46$ & & $5.69 \pm 5.18$ & & $5.67 \pm 8.02$ & \\
\hline \multicolumn{9}{|l|}{ AJCC stage } \\
\hline $1-3$ & $9.02 \pm 4.85$ & 0.607 & $21.70 \pm 12.37$ & 0.269 & $4.87 \pm 4.96$ & 0.193 & $5.61 \pm 8.11$ & 0.582 \\
\hline 4 & $9.92 \pm 4.78$ & & $16.87 \pm 10.60$ & & $7.07 \pm 3.51$ & & $4.17 \pm 4.02$ & \\
\hline \multicolumn{9}{|l|}{ Tumor Marker } \\
\hline Negative & $10.26 \pm 3.68$ & 0.534 & $14.59 \pm 8.79$ & 0.054 & $5.89 \pm 2.69$ & 0.785 & $3.53 \pm 2.32$ & 0.434 \\
\hline Positive & $9.09 \pm 5.34$ & & $23.24 \pm 12.40$ & & $5.39 \pm 5.41$ & & $5.71 \pm 8.50$ & \\
\hline
\end{tabular}

Data were expressed as Mean \pm SD. Abbreviations: NSCLC: non-small cell lung cancer; SD: standard deviation; Treg: regulatory T cells; SUV: standard uptake value; ECOG PS: Eastern Cooperative Oncology Group performance status; SCC: squamous cell carcinoma; ADC: adenocarcinoma; AJCC: American Joint Committee on Cancer.

association between other lymphocyte subsets with PFS. In addition to lymphocyte subpopulations, some other parameters were correlated with clinical outcomes (Table $6)$. The neutrophil/lymphocyte ratio (NLR) was negatively correlated to PFS $(\mathrm{HR}=2.66,95 \% \mathrm{CI}=1.01-7.05, P=$ 0.033 , Figure $2 \mathrm{~B}$ ); conversely, a positive association was observed between lymphocytes and PFS ( $\mathrm{HR}=0.42$, $95 \% \mathrm{CI}=0.17-1.03, P=0.042$, Figure 2C). Moreover, a high standard uptake value (SUV) correlated with poor survival, with a strong trend toward significance (HR $=4.14,95 \% \mathrm{CI}=0.85-20.11, P=0.051$, Figure $2 \mathrm{D})$. In terms of clinicopathologic characteristics, the Eastern Cooperative Oncology Group performance status (ECOG PS), tumor differentiation, clinical stage, and nodal stage correlated with clinical outcome of NSCLC patients (Table 6). 
Table 6: Univariate analysis of progression-free survival of 40 newly diagnosed NSCLC patients according to clinicopathologic characteristics and lymphocyte subsets

\begin{tabular}{|c|c|c|c|}
\hline Parameter & Median survival (months) & HR $(95 \% C I)$ & $\boldsymbol{P}$ \\
\hline Age $\left(\geq \mathbf{6 0} v_{s}<\mathbf{6 0}\right)$ & 17 vs 13 & $0.78(0.33-1.84)$ & 0.551 \\
\hline Sex (Male vs Female) & $13 v s \mathrm{NA}$ & $1.94(0.70-5.37)$ & 0.173 \\
\hline ECOG PS (1-2 vs $\mathbf{0})$ & $11 v s 16$ & $3.59(1.30-9.94)$ & 0.006 \\
\hline Smoking (Previous/present $v s$ Never) & 13 vs 13 & $1.33(0.54-3.27)$ & 0.521 \\
\hline SUV $\left(\geq 9.5 v_{S}<9.5\right)$ & $13 v s$ NA & $4.14(0.85-20.11)$ & 0.051 \\
\hline Histology (ADC vs SCC) & 13 vs 15 & $1.24(0.51-3.01)$ & 0.614 \\
\hline Differentiation (Poor $v s$ Well/moderate) & $8 v s 17$ & $4.91(1.70-14.15)$ & 0.001 \\
\hline Tumor Marker (Positive vs Negative) & 13 vs 15 & $1.93(0.64-5.80)$ & 0.213 \\
\hline Tumor stage (T2-4 vs T1) & $13 v s \mathrm{NA}$ & $1.70(0.57-5.03)$ & 0.312 \\
\hline Nodal stage (N1-3 vs N0) & $12 v s 16$ & $3.70(1.09-12.58)$ & 0.019 \\
\hline AJCC stage (4 vs 1-3) & 10 vs 17 & $3.21(1.37-7.50)$ & 0.003 \\
\hline Leukocyte ( $\geq$ median $v_{s}<$ median) & 13 vs 13 & $0.96(0.38-2.38)$ & 0.919 \\
\hline Lymphocyte $(\geq 1.5 v s<1.5)$ & 14 vs 11 & $0.42(0.17-1.03)$ & 0.042 \\
\hline $\operatorname{NLR}(\geq 2.54 v s<2.54)$ & 12 vs 16 & $2.66(1.01-7.05)$ & $\mathbf{0 . 0 3 3}$ \\
\hline PLR ( $\geq$ median $v s<$ median) & 13 vs 13 & $1.34(0.55-3.29)$ & 0.500 \\
\hline MLR ( $\geq$ median $v s<$ median) & 15 vs 13 & $1.21(0.50-2.94)$ & 0.655 \\
\hline Treg $(\geq 3.16 v s<3.16)$ & 12 vs 17 & $2.55(1.07-6.11)$ & 0.022 \\
\hline CD3+ $\mathbf{T}$ cells $(\geq$ median $v s<$ median $)$ & 13 vs 13 & $0.99(0.43-2.31)$ & 0.989 \\
\hline CD4+ T cells $(\geq$ median $v s<$ median) & 13 vs 13 & $1.16(0.50-2.68)$ & 0.722 \\
\hline CD8+ T cells $(\geq$ median $v s<$ median) & 17 vs 13 & $0.86(0.37-2.01)$ & 0.720 \\
\hline CD4/CD8 ratio( $\geq$ median $v s<$ median) & 13 vs 17 & $1.53(0.65-3.60)$ & 0.302 \\
\hline CD19+ B cells ( $\geq$ median $v s<$ median) & 11 vs 13 & $1.13(0.49-2.61)$ & 0.767 \\
\hline NK $(\geq$ median $v s<$ median $)$ & 13 vs 13 & $0.97(0.42-2.27)$ & 0.945 \\
\hline NKT $\left(\geq\right.$ median $v_{S}<$ median) & 11 vs 15 & $1.57(0.67-3.68)$ & 0.271 \\
\hline$\gamma \delta \mathbf{T}(\geq$ median $v s<$ median $)$ & 13 vs 15 & $1.49(0.63-3.49)$ & 0.335 \\
\hline CD8+CD28+ $\mathbf{T}$ cells $(\geq$ median $v s<$ median) & 13 vs 13 & $0.82(0.35-1.90)$ & 0.630 \\
\hline CD8+CD28- $\mathbf{T}$ cells ( $\geq$ median $v s<$ median) & 17 vs 13 & $0.67(0.29-1.55)$ & 0.325 \\
\hline
\end{tabular}

Abbreviations: NSCLC: non-small cell lung cancer; HR: hazard ratio; CI: confidence interval; Treg: regulatory T cells; SUV: standard uptake value; SCC: squamous cell carcinoma; ADC: adenocarcinoma; ECOG PS: Eastern Cooperative Oncology Group performance status; AJCC: American Joint Committee on Cancer; NLR: neutrophil/lymphocyte ratio; PLR: platelet/ lymphocyte ratio; MLR: monocyte/lymphocyte ratio.

\section{Elevated Treg cells independently predict poor PFS}

On multivariate analysis, elevated Treg cells were independently correlated with poor PFS $(\mathrm{HR}=3.76$, $95 \% \mathrm{CI}=1.38-10.22, P=0.010$, Table 7). For wellrecognized prognostic factors, the independent roles of American Joint Committee on Cancer (AJCC) stage and nodal stage were found as well.

\section{DISCUSSION}

We demonstrated that elevated Treg cells were independently associated with poor PFS in NSCLC patients received radiotherapy. In addition, NLR and the lymphocyte count were associated with tumor progression in univariate analysis. Moreover, we further validated the increased proportions of Treg cells, CD8+ T cells, and CD8+CD28- T cells and decreased CD4+ T cells and CD4/ CD8 ratios in NSCLC patients at first relapse compared to newly diagnosed patients. To our knowledge, this is the first study to validate the predictive significance of Treg cells in patients with NSCLC undergoing radiotherapy, and the expression of them in patients at first relapse.

Treg cells suppress anti-tumor activity elicited by adaptive immune system in human cancer and facilitate tumor growth [21]. Several studies have proved the prognostic and predictive significance of peripheral Treg cells in lung cancer patients undergoing surgery and 
Table 7: Multivariate analysis of progression-free survival of 40 newly diagnosed NSCLC patients

\begin{tabular}{|c|c|c|}
\hline Parameter & HR (95\%CI) & $P$ \\
\hline \multicolumn{3}{|l|}{ Treg } \\
\hline$<3.6$ & 1 & 0.010 \\
\hline$\geq 3.6$ & $3.76(1.38-10.22)$ & \\
\hline \multicolumn{3}{|l|}{ Differentiation } \\
\hline Well/moderate & 1 & 0.152 \\
\hline Poor & $2.46(0.72-8.40)$ & \\
\hline \multicolumn{3}{|l|}{ AJCC stage } \\
\hline $1-3$ & 1 & 0.005 \\
\hline 4 & 5.74(1.71-19.19) & \\
\hline \multicolumn{3}{|l|}{ Nodal stage } \\
\hline N0 & 1 & 0.004 \\
\hline N1-N3 & $8.12(1.94-33.98)$ & \\
\hline \multicolumn{3}{|l|}{ ECOG PS } \\
\hline 0 & 1 & 0.101 \\
\hline $1-2$ & $2.10(0.65-6.75)$ & \\
\hline
\end{tabular}

Abbreviations: NSCLC: non-small cell lung cancer; HR: hazard ratio; ECOG PS: Eastern Cooperative Oncology Group performance status; Treg: regulatory T cells; AJCC: American Joint Committee on Cancer.
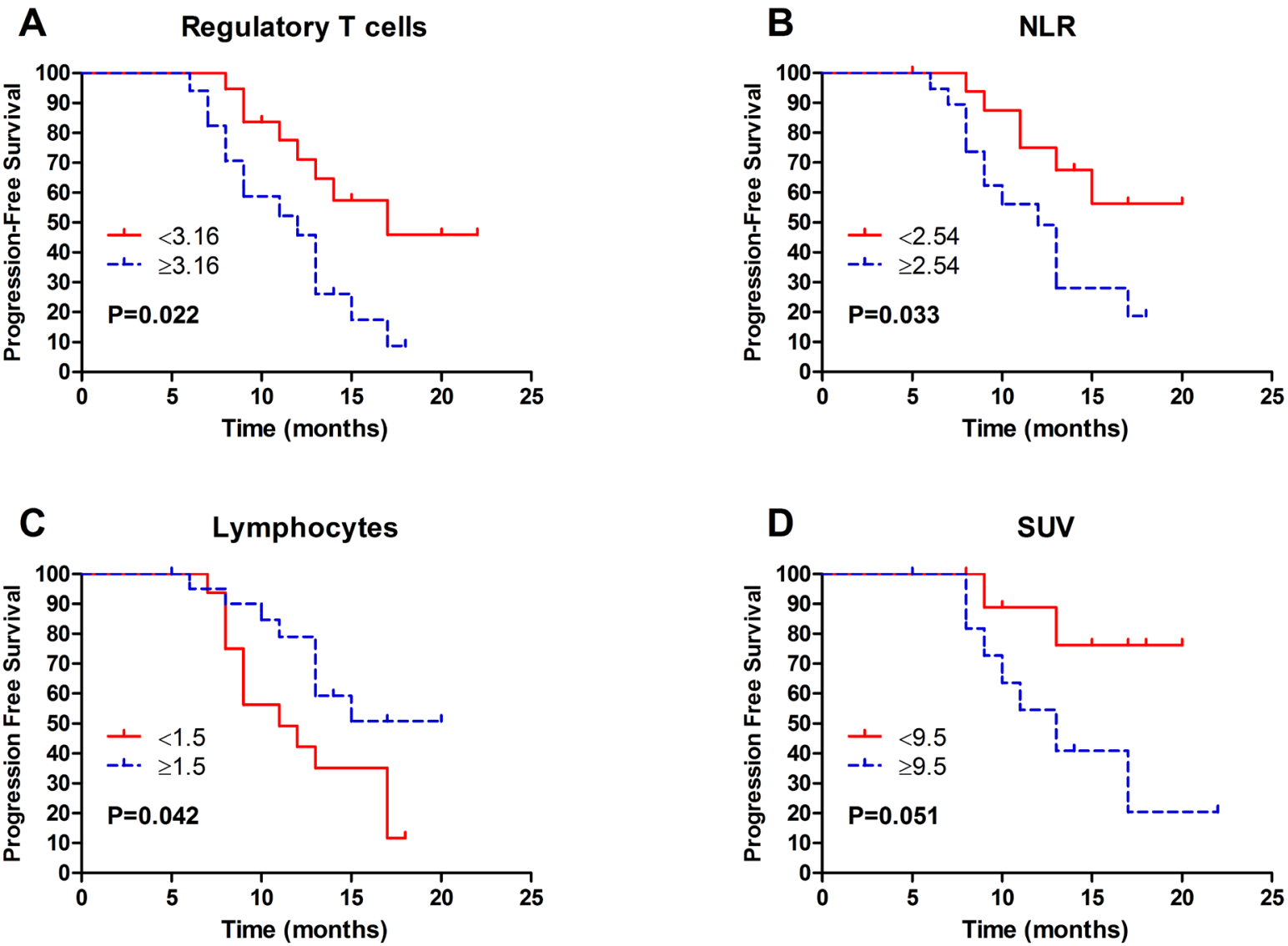

Figure 2: Kaplan-Meier of progression-free survival of 40 first diagnosed NSCLC patients according to A. Regulatory T cells; B. NLR; C. Lymphocyte count; D. SUV. Abbreviations: NSCLC = non-small cell lung cancer; NLR = neutrophil/lymphocyte ratio; $\mathrm{SUV}=$ standard uptake value. 
chemotherapy $[15,19]$. In the present study, we further confirmed its predictive role in NSCLC patients treated with radiotherapy. Elevated proportions of Treg cells indicated rapid tumor progression after radiotherapy, which may because of the suppressor function of Treg cells and their resistance to radiation [22, 23]. In addition to lymphocyte subsets, we also observed that lymphocyte counts and NLR were correlated with survival without relapse, which were consistent with published studies [24, 25]. The predictive value of NLR, platelet/lymphocyte ratio (PLR) and monocyte/lymphocyte ratio (MLR) were previously investigated in various kinds of cancer [24, 26-28]. A recent study observed that pretreatment SUV correlated with PFS in early-stage NSCLC patients treated with SBRT [29]. We found a similar trend in the 22 patients whose SUV were available, although 7 of them had been treated with conventional fraction radiotherapy.

As immunosuppressive cells, increased Treg cells were observed in various tumors and metastatic diseases, including those with NSCLC [11, 30-32]. Our findings are consistent with these observations and supported this role in patients at first relapse compared to newly diagnosed patients. In addition, we found that NSCLC patients with metastatic disease showed high level of Treg cells compared to local recurrence, which may suggest the relationship between increased Treg cells and tumor progression. However, we found no differences of other lymphocyte subsets between metastatic disease and local recurrence, which may be attributable to the limited number of patients. Our study showed decreased proportions of CD4+ T cells, CD4/CD8 ratio and CD19+ $\mathrm{B}$ cells in NSCLC patients, which is in agreement with previous studies $[12,33]$. As important immune cells that prevent tumor progression, CD8+ cytotoxic $\mathrm{T}$ lymphocytes $(\mathrm{CD} 8+\mathrm{CD} 28+\mathrm{T}$ cells) were decreased in NSCLC patients compared to healthy volunteers although it did not reach significance. CD8+CD28- T cells, exhibiting immunosuppressive role, were significantly increased in NSCLC patients, which may contribute to the increase of CD8+ T cells. Furthermore, the expression of these immune cells was confirmed in NSCLC patients at first relapse compared to patients at first diagnosis. These observations may imply the important role of adaptive immune system in cancer patients.

Younger patients, females and those with negative tumor markers presented increase of CD4+, CD8+CD28+ $\mathrm{T}$ cells, and $\mathrm{CD} 4 / \mathrm{CD} 8$ ratios in the periphery, which is consistent with a previous study [34]. However, we have not observed significant correlation between these lymphocyte subsets with tumor stage, which may attribute to the limited patients number.

There are several limitations in our study. First, the sample size is small. Second, the clinical stages of patients in our study are not homogeneous. Third, different radiation doses and fractions were used. Despite these limitations, we proved that the level of Treg cells independently predicted tumor progression. Moreover, increased Treg cells were observed in NSCLC patients at first diagnosis and at first relapse compared to healthy controls.

\section{CONCLUSIONS}

In NSCLC patients undergoing radiotherapy, the level of peripheral Treg cells may serve as a useful predictor of tumor progression, indicating that adaptive immunity is highly important for NSCLC patients. Those findings suggest that radiotherapy combined with the manipulation of Treg cells may become a successful treatment for NSCLC patients.

\section{MATERIALS AND METHODS}

\section{Patients and clinical data}

Seventy histologically confirmed NSCLC patients were prospectively enrolled in the present study, consisting of 40 newly diagnosed patients and 30 patients at first relapse. The 40 newly diagnosed patients were over 18 years old and had not received treatment for cancer before enrollment. They had no immune system related diseases, no infections, transplant history, cancer of other types, and they had not received steroid treatment before enrollment. The 30 NSCLC patients at first relapse had not received anti-tumor therapy, immunotherapy and steroid therapy for at least 3 months (exclude one who received gefitinib) before enrollment. Fourteen age-matched healthy controls were enrolled. All volunteers and patients provided informed consent. This study was approved by the Ethical Committee of The Affiliated Hospital of Academy of Military Medical Sciences.

We obtained patient characteristics from electronic records. Tumor stage was evaluated by [AJCC]-7 criteria [35]. Tumor markers for NSCLC contained squamous cell carcinoma antigen (SCC), carcinoembryonic antigen (CEA) and cytokeratinfragment (CYFRA 21-1). Tumor marker was positive when either of them was positive. NLR, PLR and MLR were collected from peripheral blood routine test.

\section{Blood samples and flow cytometry}

Fresh blood samples were collected from patients prior to anti-cancer treatment and from volunteers. Ten specific monoclonal antibodies (mAbs) against CD3 (APC and PerCP), CD4 (FITC and APC), CD8 (FITC and APC), CD16 (PE), CD19 (APC), CD25 (APC), CD28 (PE), CD56 (PE), CD127 (PE), and TCR (PE) were used to differentiate lymphocyte subsets. At the beginning, we mixed $100 \mu 1$ fresh blood with the above mAbs and 
incubated at room temperature for 15 minutes in the dark. We used FACS lysing solution (BD Biosciences, San Jose, CA, USA) to lyse red blood cells in the mix and then washed twice with phosphate buffered saline (PBS). After that, flow cytometry was used to analysis the residual white blood cells and the proportions of the lymphocyte subsets were calculated by FlowJo Version 10 data analysis software (FlowJo, Ashland, OR, USA).

Lymphocyte subpopulations were identified as follow: $\mathrm{CD} 3+\mathrm{T}$ cells (CD3+CD19-), CD4+ T cells (CD3+ CD4+CD8-), CD8+ T cells (CD3+CD8+CD4-), $\mathrm{CD} 8+\mathrm{CD} 28+\mathrm{T}$ cells $(\mathrm{CD} 3+\mathrm{CD} 8+\mathrm{CD} 28+)$, CD8+CD28- $\mathrm{T}$ cells (CD3+CD8+CD28-), Treg (CD4+CD25+CD127low), B cells (CD3-CD19+), natural killer (NK) cells (CD3-CD16+CD56+), natural killer T $(\mathrm{NKT})$ cells $(\mathrm{CD} 3+\mathrm{CD} 16+\mathrm{CD} 56+)$, gamma delta $\mathrm{T}(\gamma \delta \mathrm{T})$ cells $(\mathrm{CD} 3+\mathrm{TCR}+)$. Lymphocyte subsets were determined by the percentages of total lymphocytes.

\section{Treatment and follow up}

The 40 newly diagnosed NSCLC patients received radiotherapy alone or combined with targeted therapy/ chemotherapy concurrently or consecutively. Radiotherapy contained SBRT with 30-50 Gy/5 fractions by Cyber Knife (Accuray, Sunnyvale, CA, USA) and conventional fraction radiotherapy with 60-66 Gy/30-33 fractions using linear accelerator. Platinum-based agents were used for first-line chemotherapy; crizotinib, erlotinib, and gefitinib were used for targeted therapy.

Follow-up was ended on October 20, 2016 or the time of death of the patient. Patients were followed up regularly every 3 months after radiotherapy. The median follow-up was 12.5 months (range: 5-22 months). PFS was the primary endpoint, which calculated from the time of initial treatment to the time of first progression defined by RECIST (Response Evaluation Criteria in Solid Tumors) 1.1 [36] criteria, loss to follow-up, or death.

\section{Statistical analysis}

Proportions of lymphocyte subpopulations were expressed with mean \pm standard deviation (SD). Basic characteristics between patients at first diagnosis and relapse were compared by Chi-square and Fisher exact tests. Differences of immune parameters between the two groups of lung cancer patients and the controls were evaluated by one-way analysis of variance (ANOVA) and post-hoc multiple comparisons. The Student's $t$-test was used to determine relationships between lymphocyte subsets and patient characteristics. We employed the Kaplan-Meier analysis to estimate PFS and log-rank test to compare the survival of two groups. The Cox proportional hazards model was used to determine hazard ratios (HRs) and 95\% confidence intervals (CIs). Considering the limited number of patients, only parameters of $P<$ 0.025 in univariate analysis were included in multivariate analysis. We used the Statistical Package for Social Sciences, Version 20.0 (IBM Corporation, Armonk, NY, USA) to analyze the data. $P$ value $<0.05$ was considered statistically significant.

\section{CONFLICTS OF INTEREST}

The authors report no conflicts of interest in this work.

\section{REFERENCES}

1. Chen W, Zheng R, Baade PD, Zhang S, Zeng H, Bray F, Jemal A, Yu XQ, He J. Cancer statistics in China, 2015. CA Cancer J Clin. 2016; 66: 115-32. doi: 10.3322/caac.21338.

2. Devesa SS, Bray F, Vizcaino AP, Parkin DM. International lung cancer trends by histologic type: male:female differences diminishing and adenocarcinoma rates rising. Int J Cancer. 2005; 117: 294-9. doi: 10.1002/ijc. 21183.

3. Jaffray DA. Image-guided radiotherapy: from current concept to future perspectives. Nat Rev Clin Oncol. 2012; 9: 688-99. doi: 10.1038/nrclinonc.2012.194.

4. Chang JY, Senan S, Paul MA, Mehran RJ, Louie AV, Balter P, Groen HJ, McRae SE, Widder J, Feng L, van den Borne BE, Munsell MF, Hurkmans C, et al. Stereotactic ablative radiotherapy versus lobectomy for operable stage I nonsmall-cell lung cancer: a pooled analysis of two randomised trials. Lancet Oncol. 2015; 16: 630-7. doi: 10.1016/s14702045(15)70168-3.

5. Timmerman RD, Herman J, Cho LC. Emergence of stereotactic body radiation therapy and its impact on current and future clinical practice. J Clin Oncol. 2014; 32: 2847 54. doi: 10.1200/jco.2014.55.4675.

6. Herrera FG, Bourhis J, Coukos G. Radiotherapy combination opportunities leveraging immunity for the next oncology practice. CA Cancer J Clin. 2016. doi: 10.3322/ caac. 21358.

7. Bernstein MB, Krishnan S, Hodge JW, Chang JY. Immunotherapy and stereotactic ablative radiotherapy (ISABR): a curative approach? Nat Rev Clin Oncol. 2016; 13: 516-24. doi: 10.1038/nrclinonc.2016.30.

8. Vesely MD, Kershaw MH, Schreiber RD, Smyth MJ. Natural innate and adaptive immunity to cancer. Annu Rev Immunol. 2011; 29: 235-71. doi: 10.1146/annurevimmunol-031210-101324.

9. Appay V, van Lier RA, Sallusto F, Roederer M. Phenotype and function of human T lymphocyte subsets: consensus and issues. Cytometry A. 2008; 73: 975-83. doi: 10.1002/ cyto.a.20643.

10. Saavedra D, Garcia B, Lorenzo-Luaces P, Gonzalez A, Popa X, Fuentes KP, Mazorra Z, Crombet T, Neninger E, Lage A. Biomarkers related to immunosenescence: relationships 
with therapy and survival in lung cancer patients. Cancer Immunol Immunother. 2016; 65: 37-45. doi: 10.1007/ s00262-015-1773-6.

11. Erfani N, Mehrabadi SM, Ghayumi MA, Haghshenas MR, Mojtahedi Z, Ghaderi A, Amani D. Increase of regulatory $\mathrm{T}$ cells in metastatic stage and CTLA-4 over expression in lymphocytes of patients with non-small cell lung cancer (NSCLC). Lung Cancer. 2012; 77: 306-11. doi: 10.1016/j. lungcan.2012.04.011.

12. Karagoz B, Bilgi O, Gumus M, Erikci AA, Sayan O, Turken O, Kandemir EG, Ozturk A, Yaylaci M. CD8+CD28- cells and CD4+CD25+ regulatory T cells in the peripheral blood of advanced stage lung cancer patients. Med Oncol. 2010; 27: 29-33. doi: 10.1007/s12032-008-9165-9.

13. Ke X, Zhang S, Xu J, Liu G, Zhang L, Xie E, Gao L, Li D, Sun R, Wang F, Pan S. Non-small-cell lung cancer-induced immunosuppression by increased human regulatory $\mathrm{T}$ cells via Foxp3 promoter demethylation. Cancer Immunol Immunother. 2016; 65: 587-99. doi: 10.1007/s00262-0161825-6.

14. Schalper KA, Brown J, Carvajal-Hausdorf D, McLaughlin J, Velcheti V, Syrigos KN, Herbst RS, Rimm DL. Objective measurement and clinical significance of TILs in nonsmall cell lung cancer. J Natl Cancer Inst. 2015; 107. doi: 10.1093/jnci/dju435.

15. Tao H, Mimura Y, Aoe K, Kobayashi S, Yamamoto H, Matsuda E, Okabe K, Matsumoto T, Sugi K, Ueoka H. Prognostic potential of FOXP3 expression in non-small cell lung cancer cells combined with tumor-infiltrating regulatory T cells. Lung Cancer. 2012; 75: 95-101. doi: 10.1016/j.lungcan.2011.06.002.

16. Kinoshita T, Muramatsu R, Fujita T, Nagumo H, Sakurai T, Noji S, Takahata E, Yaguchi T, Tsukamoto N, Kudo-Saito C, Hayashi Y, Kamiyama I, Ohtsuka T, et al. Prognostic value of tumor-infiltrating lymphocytes differs depending on histological type and smoking habit in completely resected non-small cell lung cancer. Ann Oncol. 2016. doi: 10.1093/annonc/mdw319.

17. Zeng DQ, Yu YF, Ou QY, Li XY, Zhong RZ, Xie CM, Hu QG. Prognostic and predictive value of tumor-infiltrating lymphocytes for clinical therapeutic research in patients with non-small cell lung cancer. Oncotarget. 2016; 7: 13765-81. doi: 10.18632/oncotarget.7282.

18. Feng W, Li Y, Shen L, Cai XW, Zhu ZF, Chang JH, Xiang JQ, Zhang YW, Chen HQ, Fu XL. Prognostic value of tumor-infiltrating lymphocytes for patients with completely resected stage IIIA(N2) non-small cell lung cancer. Oncotarget. 2016; 7: 7227-40. doi: 10.18632/ oncotarget.6979.

19. McCoy MJ, Nowak AK, van der Most RG, Dick IM, Lake RA. Peripheral CD8(+) T cell proliferation is prognostic for patients with advanced thoracic malignancies. Cancer Immunol Immunother. 2013; 62: 529-39. doi: 10.1007/ s00262-012-1360-z.

20. Kotsakis A, Koinis F, Katsarou A, Gioulbasani M,
Aggouraki D, Kentepozidis N, Georgoulias V, Vetsika EK. Prognostic value of circulating regulatory $\mathrm{T}$ cell subsets in untreated non-small cell lung cancer patients. Sci Rep. 2016; 6: 39247. doi: 10.1038/srep39247.

21. Josefowicz SZ, Lu LF, Rudensky AY. Regulatory T cells: mechanisms of differentiation and function. Annu Rev Immunol. 2012; 30: 531-64. doi: 10.1146/annurev. immunol.25.022106.141623.

22. Schuler PJ, Harasymczuk M, Schilling B, Saze Z, Strauss L, Lang S, Johnson JT, Whiteside TL. Effects of adjuvant chemoradiotherapy on the frequency and function of regulatory $\mathrm{T}$ cells in patients with head and neck cancer. Clin Cancer Res. 2013; 19: 6585-96. doi: 10.1158/10780432.ccr-13-0900.

23. Persa E, Balogh A, Safrany G, Lumniczky K. The effect of ionizing radiation on regulatory $\mathrm{T}$ cells in health and disease. Cancer Lett. 2015; 368: 252-61. doi: 10.1016/j. canlet.2015.03.003.

24. Cannon NA, Meyer J, Iyengar P, Ahn C, Westover KD, Choy H, Timmerman R. Neutrophil-lymphocyte and platelet-lymphocyte ratios as prognostic factors after stereotactic radiation therapy for early-stage non-small-cell lung cancer. J Thorac Oncol. 2015; 10: 280-5. doi: 10.1097/ jto.0000000000000399.

25. Shaverdian N, Veruttipong D, Wang J, Schaue D, Kupelian $\mathrm{P}$, Lee P. Pretreatment Immune Parameters Predict for Overall Survival and Toxicity in Early-Stage Non-SmallCell Lung Cancer Patients Treated With Stereotactic Body Radiation Therapy. Clin Lung Cancer. 2016; 17: 39-46. doi: 10.1016/j.cllc.2015.07.007.

26. Cummings M, Merone L, Keeble C, Burland L, Grzelinski M, Sutton K, Begum N, Thacoor A, Green B, Sarveswaran J, Hutson R, Orsi NM. Preoperative neutrophil:lymphocyte and platelet:lymphocyte ratios predict endometrial cancer survival. Br J Cancer. 2015; 113: 311-20. doi: 10.1038/ bjc.2015.200.

27. Liu C, Huang Z, Wang Q, Sun B, Ding L, Meng X, Wu S. Usefulness of neutrophil-to-lymphocyte ratio and plateletto-lymphocyte ratio in hormone-receptor-negative breast cancer. Onco Targets Ther. 2016; 9: 4653-60. doi: 10.2147/ ott.s106017.

28. Lan H, Zhou L, Chi D, Zhou Q, Tang X, Zhu D, Yue J, Liu B. Preoperative platelet to lymphocyte and neutrophil to lymphocyte ratios are independent prognostic factors for patients undergoing lung cancer radical surgery: A single institutional cohort study. Oncotarget. 2016 Nov 11. doi: 10.18632/oncotarget.13312. [Epub ahead of print].

29. Horne ZD, Clump DA, Vargo JA, Shah S, Beriwal S, Burton SA, Quinn AE, Schuchert MJ, Landreneau RJ, Christie NA, Luketich JD, Heron DE. Pretreatment SUVmax predicts progression-free survival in early-stage non-small cell lung cancer treated with stereotactic body radiation therapy. Radiat Oncol. 2014; 9: 41. doi: 10.1186/1748-717x-9-41.

30. Wolf AM, Wolf D, Steurer M, Gastl G, Gunsilius E, Grubeck-Loebenstein B. Increase of regulatory T cells in 
the peripheral blood of cancer patients. Clin Cancer Res. 2003; 9: 606-12.

31. Kono K, Kawaida H, Takahashi A, Sugai H, Mimura K, Miyagawa N, Omata H, Fujii H. CD4(+)CD25high regulatory $\mathrm{T}$ cells increase with tumor stage in patients with gastric and esophageal cancers. Cancer Immunol Immunother. 2006; 55: 1064-71. doi: 10.1007/s00262-0050092-8.

32. Wang ZK, Yang B, Liu H, Hu Y, Yang JL, Wu LL, Zhou $\mathrm{ZH}$, Jiao SC. Regulatory T cells increase in breast cancer and in stage IV breast cancer. Cancer Immunol Immunother. 2012; 61: 911-6. doi: 10.1007/s00262-011-1158-4.

33. Nakamura H, Saji H, Ogata A, Hosaka M, Hagiwara M, Kawasaki N, Konaka C, Kato H. Immunologic parameters as significant prognostic factors in lung cancer. Lung Cancer. 2002; 37: 161-9.
34. Nakamura H, Kawasaki N, Hagiwara M, Saito M, Konaka $\mathrm{C}$, Kato H. Cellular immunologic parameters related to age, gender, and stage in lung cancer patients. Lung Cancer. 2000; 28: 139-45.

35. Edge SB, Compton CC. The American Joint Committee on Cancer: the 7th edition of the AJCC cancer staging manual and the future of TNM. Ann Surg Oncol. 2010; 17: 1471-4. doi: 10.1245/s10434-010-0985-4.

36. Eisenhauer EA, Therasse P, Bogaerts J, Schwartz LH, Sargent D, Ford R, Dancey J, Arbuck S, Gwyther S, Mooney M, Rubinstein L, Shankar L, Dodd L, et al. New response evaluation criteria in solid tumours: revised RECIST guideline (version 1.1). Eur J Cancer. 2009; 45: 228-47. doi: 10.1016/j.ejca.2008.10.026. 